\title{
IPv6 and Homenetworking
}

\author{
Tayeb Ben Meriem \\ European IPv6 Task Force Steering Committee member \\ tayeb.benmeriem(a)orange-ftgroup.com
}

\section{Introduction}

This submission focuses on highlighting the main benefits that IPv6 could bring to home networking services, based on wired and wireless architectures. Particularly wireless sensor networks (WSN) from the European IPv6 Task Force Steering Committee 's standpoint.

This work has been carried out within the "Applications" topic. The main objective being to identify the trends, for decision makers, from a business and technical perspective, as well as the role of applications, as an enabler in the acceleration of IPv6 deployment.

The chosen approach was to focus on a few relevant applications (Multi-play, home networking, M2M/RFID, Communicating Object Networks, Sensor Networks, Social Networks based on Web2.0, Emergency \& Crisis, Transportation, Push applications).

These applications should be coupled to the access networks (Fixed \& Mobile: xDSL, Cable, FTTH, ETTX, WIMAX, 3G, HSPA, DVB$\mathrm{H} / \mathrm{T} / \mathrm{S} / \mathrm{SH}, .$.$) . These applications are projected to be the potential "gold-$ mine" of revenue growth in the coming years for ISPs and associated partners in the access value chain (content providers, vendors, software providers, handsets manufactures, home electronic devices manufacturers ..) [Euv6TF].

From a broadband penetration perspective, at least 6 European countries are ranked on the top 10 list. This suggests that Europe is ideally positioned to take advantage of applications \& services deployment using IPv6. This is a significant outcome from the "eEurope 2005"[eEurope2005] initiative, with over $180 \mathrm{M} €$ invested in IPv6 projects during the last 6 years.

The European Commission's " $\mathrm{i}-2010 "$ initiative [i-2010] is more focusing on concrete objectives hence, it is the foundational "next step" for IPv6 deployment and associated market development.

Therefore, we believe that Broadband access services and IPv6 technology presents a unique opportunity for all the value chain actors in fixed and mobile access networks, for developing the home applications market.

Please use the following format when citing this chapter:

Meriem. T. B., 2007, in IFIP International Federation for Information Processing, Volume 256, IIome Networking. Al Agha, K., Carcelle. X.. Pujolle, G., (Boston: Springer), pp. 267-273. 
Within the European IPv6 Task Force Steering Committee work plan, the question related to Home network is a key topic.. It is for this reason, a study surveying the Home networking ecosystem from technical, industry, business and standardisation perspective has been carried out. [EUv6TF]

For the latter item, since 2004, the IPv6 Steering Committee has been working alongside the major fora addressing the issue of Home networking. Liaisons with CENELEC [CENELEC], SmartHouse Forum, HGI [HGI] , DLNA [DLNA], North American IPv6 Task Force [NAv6TF] and the IPv6 promotion Council China [IPv6China] were established.

Home IP services integrate Voice over IP or more generally Triple plays, on line gaming or remote controlling for instance. Since an ISP has to be able to provide a wide area of services to its customer, it has to think about the right solution for such networks. Indeed, the current solutions based on IPv4 protocol suffer limitations:

One of the requirements of IP home networks is to facilitate the installation and the configuration of devices.

The first step is to configure the device with an IP address. IPv4 protocols do not provide any stateless configuration service that permits a device to establish an external connection.

The following are the main technical challenges we identified that should be overcome in order to develop an attractive home network.

- How to allow users to connect and communicate regardless of device type, manufacturer, or network technology?

- How to do name resolution in the absence of DNS?

- How to auto-configure services?

- How to bridge between different network technologies (e.g. Bluetooth, $802.11 \mathrm{~b}$,)?

- How feasible is it to implement IPsec?

- How to implement QoS?

- Multicast?

- Multi-homing?

- Transition \& Interoperability?

Therefore, it appears that IPv6 can fill in the gap! 


\section{How can IPv6 bring to Homenetworking: IPv6 Feature Set}

IPv6 provides features that allow the set up of networks very easily and integrates characteristics that ease the deployment of value added services such as multicast or mobility.

Moreover, the security of communications and integrity of data in the home network, as well as authentication and confidentiality will be improved with the new version of IP protocol, thanks to the integration of IPsec within the protocol.

The auto-configuration of all home devices (plug \& play manner) is also one of major features of IPv6. It is this auto-configuration that places the Internet within everyone's reach: "I hook up my terminal; it obtains an IP address automatically and is immediately operational" (no configuration needed).

IPv6's ability to introduce new services on a larger scale and at less cost through the simple integration of new features, without the need for additional equipment is another advantage it has over IPv4. The capacity for multi-homing (a client can be hooked up to several ISPs) through the IETF SHIM6 protocol (Site Multihoming by IPv6 Intermediation) [Shim6] provides a more efficient method of multihoming than what's available with IPv4.

From a user perspective, the Home should not be seen as an isolated area, but it should be included in its entire social and business environment. That means the home should be extended to the car, to the office, to the airport, to the train, as in real life.

Thus, this user case can be studied from a convergence perspective. Technically speaking, we need to implement IPv6 mobile protocols allowing host mobility (MIPv6) as well as network mobility (Nemo)along with the target operators network architecture NGN/IMSv6-based (New Generation Network/Internet Multimedia Subsystem)[NGN][IMS]. Indeed, this NGN access network will be directly connected to the home networks and the associated huge number of devices. 
The IMS control plane SIPv6-oriented [SIP] will be at the heart of this architecture. From a services perspective, the application plane based on so called "Applications servers" or (SA) will open up new opportunities of development of advanced applications that the home market should be the main beneficiary.

The home networking cost model is another main challenge for user acceptance. It is linked to CAPEX \& OPEX (the installation cost and operation/management cost). This cost can be reduced or overcome by eliminating cumbersome wired architecture. Thus, IPv6-based wireless sensor networks architectures will be progressively introduced in the home, reducing the CAPEX consequently. Moreover, the connectivity of the Home to Internet will allow the user to remotely manage its home services and reducing significantly the associated OPEX. .

Therefore, such cost effective solutions will open up new opportunities for the home networking market.

Consequently the effort is focusing on the wireless sensor home network. Indeed, they will raise new issues pertaining to IPv6 implementation from a sensor nodes constraints and routing perspective

Home sensors devices and sensor nodes at large, are constrained devices in terms of energy and CPU and the main challenge is how to port these constrained communication sensors to IPv6. Some platforms used by the academic world have been recently developed, with first commercial products made available (Archrock,...) .

Regarding routing angle, WSNs nodes in most cases have limited or no mobility whereas ad hoc network nodes are mostly mobile; therefore, ad hoc routing protocols [MANET] such as AODV: Ad-hoc On Demand Vector Routing, DSDV: Destination Sequenced Distance Vector, DSR: Dynamic Source Routing, OLSR: Optimized Link State Routing... are not adapted to the WSNs.

This is why; new protocols must be designed for this purpose.

In this context, IETF working Groups are actively working on these topics such as Manemo [Manemo], (IEEE 802.15.4,...).

\section{How to move to IPv6?}


According to the recent IANA's forecast [ ], IPv4 address blocks will be unavailable from 2012. We will all have to deal with the consequences.

So, the question for the Internet Community is no longer - "should we move to IPv6?" but rather, " how do we prepare for IPv6, ?" and we must start now!

Two key points lead to this situation.

1. The management of IPv4 addresses blocks in one hand and

2. The huge development and popularization of Internet services

The initial rules of IPv4 address attribution were established without proper controls.

Efficient measures were put into place during the 1990s:

- Decentralised management structures

- Management rules with economy of use in mind

- New address networking techniques (CIDR "Classless Inter-Domain Routing", NAT, etc.).

These measures only apply to new requests. Consequently, they do not place most of the spaces attributed before 1994, into jeopardy. This was the year that the regional registers appeared.

IPv6's coming of age, offers the opportunity to once again place all the players on an equal footing. The management of IPv6 address blocks is also based on the five national registers that manage the IPv4 address blocks (RIPE for Europe, APNIC for Asia/Pacific, ARIN for the United States and Northern America, LACNIC for Latina America and Caribbean, and AFRINIC for Africa).

For this reason, the question of IPv6 should be seen at a global level.

From a business standpoint, this large addressing capability will allow the development of flexible solutions to the mass markets such as Domestic devices, general public electronics, etc. as well as those of ADSL access networks, third generation mobile networks, communicating transportation (planes, trains, buses and cars), smart objects (sensor networks, Machine to Machine, etc.), audio or video-type interpersonal services (PtoP), aeronautics and military communications. 


\section{Conclusion}

The current situation of the Internet can be described by two separate worlds, the "actual Internet", IPv4 based and its slow migration to IPv6, in one hand, and the "Internet of things" or M2M or communicating objects not IP based, on the other

Therefore, the challenge for the coming years is how to bridge these two worlds in order to include the huge number of devices and sensor networks in the global Internet.

IPv6 can be an appropriate instrument to help the development of this market in which home networking cases are the main driver. 


\section{References:}

[Euv6TF]: http://www.ec.ipv6tf.org/PublicDocuments/Press_Release_IPv6TF.pd [eEurope2005]:

hittp://europa.eu.int/information_society/eeurope/news_library/documents/eeurope2005/eeu rope 2005 en.pdf

[i-2010]: ec.europa.eu/i2010

[CENELEC]:

http://www.cenelec.org/Cenelec/CENELEC+in+action/News+Centre/Press+releases/Smart +House+IPv6+PR.htm

HGI]: www homegatewayinitiative.org

[DLNA]: http://www.dlna.org

[NAv6TF]: www.nav6tf.org

[IPv6China]:: ww.conference.cn/ipv6/2007/Overview.asp

[Shim6]: www.ietf.org/html.charters/shim6-charter.html

|NEMO|: Ref: RFC 3963 - Network Mobility (NEMO) Basic Support Protocol

[NGN]: ITU-T WTSA-04

[IMS]: ETSI IMS TISPAN

[SIP|: http://www freebit.com/english/index.html)

[Archrock]: www.archrock.com

[MANET]:www.ietf.org/html.charters/manet-charter.html

[Manemo]: draft-culler-rl2n-routing-reqs-01, draft-brandt-rl2n-home-routing-reqs-01),

[IANA]: www.iana.org 